\title{
Fundamental Particle and Forces
}

\author{
Bhumi Raj Sharma (Gautam) \\ Prithivi Narayan Campus Pokhara, Tribhuvan University Nepal. \\ bhysics@gmail.com
}

\begin{abstract}
The study of particle physics is indispensable not only to understand different branch of science but also to unleash the most profound mysteries in natures such as the mystery of the origin of the Universe. Fundamental particles and their interactions in nature come under the study of Particle Physics, which studies the physical processes that occurs at scales even smaller than atomic nuclei. This article introduces elementary particles and forces, and then focuses on a few promising and interesting area of the particle physics.
\end{abstract}

Keywords: Quarks, Neutrinos, Colour, Gluons, Antiparticles, Flavors

\section{INTRODUCTION}

People have a propensity to wonder what things are made of -- at least since the Greeks, and undoubtedly much earlier. Aristotle, following the Plato, thought that matter was based on four fundamental substances: earth, air, and fire, as well as water. In fact, Aristotle added a fifth essence, "quintessence" that supposedly resided in the celestial world. Other Greek philosophers took a different approach. Instead of trying to identify the essences of matter, they were content to view matter in terms of the ability to divide it into smaller and smaller units until some ultimate indivisible unit they called the "atom" was reached. Leucippus of Miletus and especially his student Democritus are recognized for promoting this view. Leucippus and Democritus, of course, weren't that far off, in the view of modern physics. Except that what we now call atoms are known not to be indivisible. In the early 20th century it was recognized that atoms are made of even smaller particles -- electrons, protons, and neutrons. Somewhat later, in the 1960s, it became apparent that protons and neutrons were themselves composed of smaller particles -- quarks -- though electrons remained indivisible. There are now various good reasons to think that electrons and quarks really are fundamental and indivisible.

\section{FUNDAMENTAL PARTICLES}

Around 1970, physical theories of the fundamental nature of matter began to converge on what is now called the "standard model" or particle physics. According to this model there are the "elementary particles" that make up matter, which are electrons, neutrinos, and quarks.
The uncharged and almost massless neutrinos exist in great abundance but hardly interact with normal matter at all. Nevertheless, electrons and neutrinos are closely associated, and each has relatives which differ only in mass. Likewise, there are several different types of quarks besides the two that occur in ordinary matter. Each particle has corresponding anti-particle, which differs in electrical charge except for neutrinos, which are uncharged. Neutrions don't interact with the matter that makes up our bodies, there are many billions of neutrinos bombarding our bodies at any given second. Neutrinos are incredibly numerous. Some originated during the Big Bang, while others arise from the impact of cosmic rays with the upper atmosphere and even radioactive decay of elements in the Earth's crust. They come in three "flavors" stated as tau, electron, and muon. Earlier experiments investigating the muon neutrino earned the Nobel Prize in Physics in 1988.

In 1998, a Japanese team under the leadership of Dr. Kajita found that muons can actually flip flavors on their own.

Shortly after, a Canadian team proved that neutrinos from the Sun are undergoing a similar flavor-switch on their way to Earth. It showed that neutrinos must have mass and the tiny masses of neutrinos could sum to weight more than all the stars in the universe. The two teams were awarded the 2015 Nobel Prize in Physics. The two recipients were Arthur McDonald of Canada's Queens University, and Takaaki Fajita of Japan's University of Tokyo.

Ordinary matter is made of from electrons and quarks. Quarks are the constituents that build protons, neutrons and the nuclei. However, it is not possible to produce free quarks. Only aggregates of quarks, two or three, can exist freely such as the proton. Quarks have electric charges which 
are a fraction of the proton's, $-1 / 3$ or $+2 / 3$, a strange feature which has not yet been explained. Each quark also has a special property like its electric charge, which is quantized, that is, it can only take on certain values. This property is called colour charge, owing to its similarity to the concept of colour. Quarks can carry the color charges red, blue or green. For every quark there is an antiquark in the same way as the electron has an antiparticle, the positron. Antiquarks have the color charges antired, antiblue or antigreen. Groups of quarks, which can exist freely, are color neutral. The three quarks in the proton $\mathrm{u}, \mathrm{u}$ and $\mathrm{d}$ have different colour charges so that the total colour charge is white or neutral.

\section{FUNDAMENTAL FORCES}

Along with the fundamental particles there are also fundamental forces, which are manifested in interactions between the particles. The first force is electromagnetic. The second force is gravity. The remaining forces are the "weak" force and the "strong" force, which are observable only in interactions between particles and not a part of direct everyday experience.

For each of these force types, there are additional particles which are said to "carry" or "mediate" the force. That is, the force is regarded as resulting from an exchange of these mediating particles, which are of a type referred to as "bosons". These particles are called "photons", "gravitons", "gluons", and W or Z bosons -- corresponding respectively to the electromagnetic, gravitational, strong, and weak forces. The weak force is much weaker than electromagnetic force at typical distances within an atomic nucleus but is still stronger than gravity, and has a short range, so has very little effect as a force, but has the property of changing one particle into another. It can cause a down quark to become an up quark, and in the process release a high energy electron and electron anti-neutrino. This is known as beta decay, a form of radioactivity.

On the other hand, the strong force, one of nature's four basic forces or, color interaction, acts between the quarks. In the same way as electrically neutral molecules can form bonds through the attraction between their positive and negative parts the exchange of force between protons and neutrons in the nucleus occurs through the colour forces that leak out from their quarks and force-carrying particles. The force between quarks is carried by gluons (from the word 'glue'), which, like photons, lack mass. Gluons, however, in contrast to photons, also have the property of colour charge, consisting of a colour and an anticolour. This property is what makes the colour force so complex and different from the electromagnetic force. Unlike gravity and electromagnetism, the strong force increases with increasing distance: It is loosely similar to the restoring force of an extended spring.

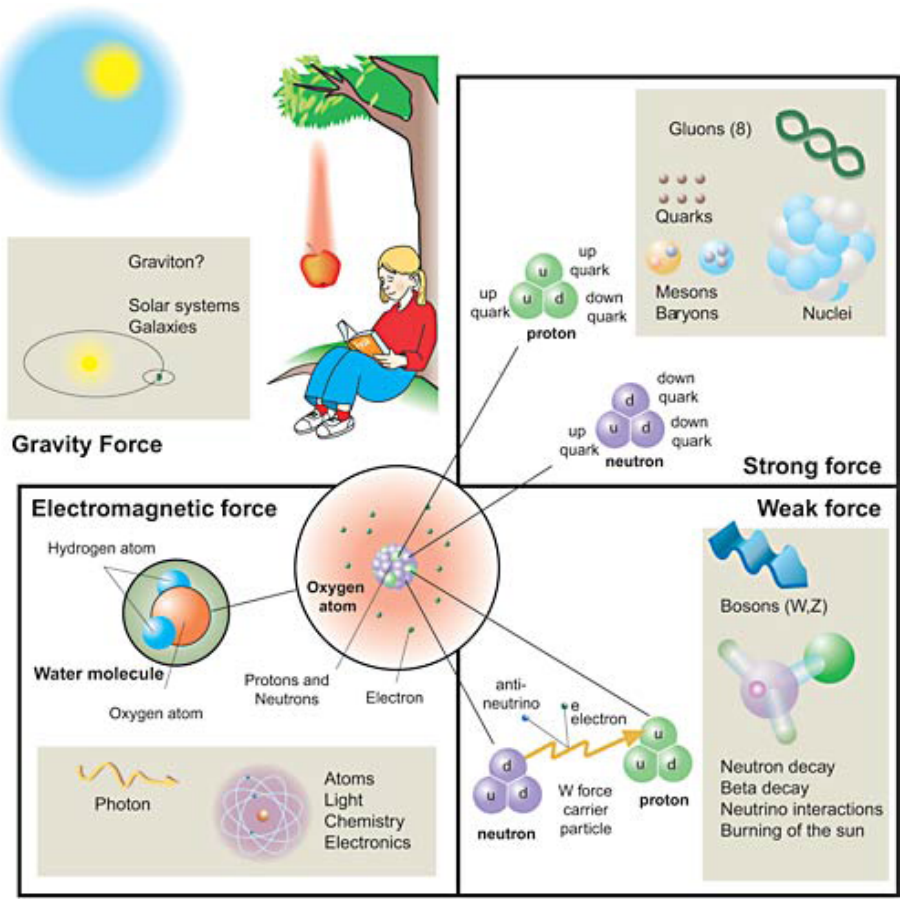

Fig. 1. The four interactions of nature, their force carrying particles and the phenomena or particles affected by them.

\section{CONCLUSION}

The particle physics is not only the subject of interest among researchers and students of physics but also it is promising field of physics which satisfies human curiosity. Besides, neutrinos could possibly be a very telling way of looking into the universe, since they can easily pass all the way through and come out the other side of the earth. They'll need to be affected by core of the Earth in some way to be useful for deriving information about the core of the Earth itself or, significantly, inside stars. Finally, there is hypothesized to exist one more type of particle, the "Higgs boson", jokingly referred to as the "God particle," which has not yet been definitively observed, but is absolutely essential to the theory. The Higgs particle is the mechanism by which the non-massless particles acquire their mass. It is currently 
the most sought-after "fugitive" in all of physics. Although it might seem that the fact it hasn't shown up yet leaves the standard model in a rather precarious position, the success of the model in predicting all observations to date makes physicists very sure that the Higgs particle exist.

\section{REFERENCES}

1. Quigg, C. (1985). Elementary particles and forces. Scientific American, 252(4), 84-94.

2. Georgi, H., \& Glashow, S. L. (1974). Unity of all elementary-particle forces. Physical Review Letters, 32(8), 438.

3. Yuan, Y. H. (2005). On the study of neutrino properties. arXiv preprint physics/0512131.

4. Ho-Kim, Q., \& Pham, X. Y. (2013). Elementary particles and their interactions: concepts and phenomena. Springer Science \& Business Media.

5. http://www.extremetech.com/extreme/215727why-a-flipped-neutrino-just-won-the-nobel-prize

6. http://www.openquestions.com/oq-hep.htm

7. https://www.nobelprize.org/nobel_prizes/physics/ laureates/2004/popular.html

8. https://www.gotquestions.org/God-particle.html 